Proyecciones

Vol. 22, No 3, pp. 237-242, December 2003.

Universidad Católica del Norte

Antofagasta - Chile

\title{
CLASSES OF FORMS WITT EQUIVALENT TO A SECOND TRACE FORM OVER FIELDS OF CHARACTERISTIC TWO*
}

\author{
ANA CECILIA DE LA MAZA \\ Universidad de Talca, Chile
}

\begin{abstract}
Let $F$ be a field of characteristic two. We determine all nonhyperbolic quadratic forms over $F$ that are Witt equivalent to a second trace form.
\end{abstract}

Mathematics Subject Classification : Primary 11 E 04; Secondary 11 E 81

Key Words: Trace form, characteristic two

\footnotetext{
${ }^{*}$ This work was supported by Fondecyt grants $\mathrm{N}^{\circ} 1010205$, and by Programa formas extremas y representación de formas cuadráticas, Universidad de Talca
} 


\section{Introduction}

Let $E / F$ be a finite separable field extension. We define the trace form for this extension by $q(x)=\operatorname{tr}_{E / F}\left(x^{2}\right)$. When the characteristic of $F$ is not equal to 2 , the trace form $(E, q)$ is non-degenerate. However, if the characteristic is 2 then $(E, q)$ is degenerate and splits as [1] $\perp V$, with $V$ totally isotropic. It is therefore natural to introduce a modified "second trace form". To this end one considers for each $a \in E$ its characteristic polynomial

$$
p(x, a)=x^{n}-T_{1}(a) x^{n-1}+T_{2}(a) x^{n-2}+\cdots+(-1)^{n} T_{n}(a)
$$

(whence $T_{1}(a)=\operatorname{tr}_{E / F}(a)$ and $T_{n}(a)$ is the norm of $a$ ). It is clear that $\left(E, T_{2}\right)$ is a quadratic form. When the degree $n$ of the extension is odd this form is necessarily singular. To arrive at a non-degenerate form, two methods have been proposed in the literature. One method, due to Bergé and Martinet $[\mathrm{BM}]$, increases the dimension of the space by 1 using the étale $F$-algebra. The other method, due to Revoy [R], reduces the dimension of the space by 1 . In this note we will adopt the second method and call such forms 2-trace forms.

We consider the following problem: Which elements $[q] \neq 0$ of the Witt-group $W_{q}(F)$ are represented by 2-trace forms?. Our theorem 3 fully answers this question. Moreover, we will partially answer the same question for $[q]=0$ (see Prop. 1 and Prop. 2). For fields of characteristic not equal to 2 this problem seems quite more complicated: for partial results concerning generic fields one may consult $[\mathrm{CP}]$ and $[\mathrm{EHP}]$; a complete solution for Hilbertian fields is given in [Sch], [KS] and [Wat].

\section{The second trace form}

As we remarked in the introduction, there are two ways to define a second trace form. In this section we will prove that in fact the corresponding forms are Witt equivalent.

Let $E / F$ be a finite separable field extension. The second trace form $T_{E / F}$ of the extension $E / F$ was defined by Revoy $[\mathrm{R}]$ as $\left(E, T_{2}\right)$ if the degree $[E: F]$ is even, and as $\left(E_{0}, T_{2}\right)$ if the degree is odd, where $T_{1}, T_{2}$ are given by (1.1) and $E_{0}=\operatorname{Ker} T_{1}$. It is important to remark that the bilinear form $b_{q}$ associated to $T_{E / F}$ satisfies the following relations:

$$
b_{q}(x, y)=T_{2}(x+y)-T_{2}(x)-T_{2}(y)=T_{1}(x y)-T_{1}(x) T_{1}(y)
$$




$$
T_{1}\left(x^{2}\right)=\left(T_{1}(x)\right)^{2} \quad \text { and } \quad b_{q}\left(x^{2}, y^{2}\right)=b_{q}(x, y)^{2}
$$

On the other hand, Bergé and Martinet defined in $[\mathrm{BM}]$ the second trace form as Revoy if the degree $[E: F]$ is even and as $\left(E \times F, T_{2}\right)$ if not.

Theorem 1. The Revoy form and the Bergé-Martinet form are Witt equivalent.

Proof. If $[E: F]$ is odd, then the form $\left(E \times F, T_{2}\right)$ of Bergé and Martinet [BM, p. 14] splits as follows $(F(1,0)+F(0,1)) \perp E_{0} \times\{0\}$. Since $F(1,0)+$ $F(0,1)$ is an hyperbolic plane, the claim follows immediately.

\section{2-algebraic forms}

In this section we determine all non-hyperbolic quadratic forms over $F$ that are Witt equivalent to some second trace form. Furthermore we give fields where hyperbolic forms are Witt equivalent to a second trace form.

Theorem 2. Let $E / F$ be a finite separable field extension, with $[E: F]=$ $2 n+1$ or $[E: F]=2 n$. Then $T_{E / F}=(n-1) \mathbf{H} \perp[1, a]$, for some $a \in F$.

Proof. The assertion is deduced from Theorem 1 above and Theorem 3.5 in [BM, p. 13-14]. In order to illustrate the ideas, we will give the proof in case $[E: F]=2 n+1$.

Since $\left(E_{0}, T_{2}\right)$ is nonsingular, there exists a symplectic basis $\left\{e_{i}, f_{i}\right\}_{1 \leq i \leq n}$ of $E_{0}$. Hence for each $1 \leq i \leq n$ there exists $x_{i}, y_{i} \in F e_{i}+F f_{i}$ such that $T_{2}\left(x_{i}\right) \neq 0$ and $b_{q}\left(x_{i}, y_{i}\right)=1$ (note that $[0,0]=[1,0]$ [Sa1, p. 150]).

Put $e_{i}^{\prime}:=T_{2}\left(x_{i}\right)^{-1} x_{i}^{2}$ and $f_{i}^{\prime}:=T_{2}\left(x_{i}\right) y_{i}^{2}$. We have by (2.2) that $e_{i}^{\prime}, f_{i}^{\prime} \in$ $E_{0}$ and $b_{q}\left(e_{i}^{\prime}, f_{i}^{\prime}\right)=1$. Since $T_{2}\left(e_{i}^{\prime}\right)=1$, we obtain a symplectic basis such that the quadratic form decomposes as follows

$$
T_{E / F}=\left[1, a_{1}\right]+\left[1, a_{2}\right]+\cdots+\left[1, a_{n}\right],
$$

where $a_{i}=T_{2}\left(f_{i}^{\prime}\right)$. Using the relation $[1, b]+[1, c] \cong[1, b+c]+\mathbf{H}$ over $F$, we obtain $T_{E / F} \cong(n-1) \mathbf{H}+[1, a]$ with $a \equiv a_{1}+a_{2}+\cdots a_{n} \wp(F)$, where $\wp(F):=\left\{x^{2}+x \mid x \in F\right\}$.

A form $T$ over $F$ is called 2-algebraic if it is Witt equivalent to some second trace form. 
Corollary 1. A non hyperbolic quadratic form $(V, q)$ over $F$ is 2-algebraic if and only if $(V, q)=r \mathbf{H} \perp\left(V_{a}, q_{a}\right)$, with $V_{a}$ an anisotropic plane representing 1.

Proof: $\Rightarrow$ ) Is clear by Theorem 2 .

$\Leftarrow)$ Let $(V, q)=r \mathbf{H} \perp\left(V_{a}, q_{a}\right)$, with $\left(V_{a}, q_{a}\right)$ 2-dimensional anisotropic representing 1 . Then we can rewrite $\left(V_{a}, q_{a}\right)=[1, b]$, where $b \notin \wp(F)$. Let $E=F(\alpha)$ with $\alpha \in \bar{F}$ and $\alpha^{2}+\alpha+b=0$. We have that $\left(E, T_{E / F}\right)=[1, b]$.

Example 1. Let $F=\mathbf{F}_{2}(a)$ and $E=F(b)$, where $a^{2}+a+1=0$ and $b^{3}+b+a=0$. Then $T_{E / F}=[1, a] \neq[1,1]$.

In fact, using (2.1), we see that $\left\{b^{2},(1+a) b\right\}$ is a symplectic basis for $E_{0}=\operatorname{Ker} T_{1}$. Since $p(x,(1+a) b)=x^{3}+a x+a, 1 \in \wp(F)$ and $a \notin \wp(F)$, we obtain the form $\left(E_{0}, T_{2}\right)=[1, a] \neq[1,1]$.

Corollary 2. If a non hyperbolic quadratic form $(V, q)$ over $F$ is 2-algebraic then there exists a quadratic extension field $E$ of $F$ such that the extension $\left(V \otimes_{F} E, q_{E}\right)$ is hyperbolic.

Proof: See the proof of Theorem3 and note that $[1, b]=\mathbf{H}$ over $E=F(\alpha)$, with $\alpha^{2}+\alpha+b=0$ (see [Sa1, p. 150]).

Theorem 3. Let $F=\mathbf{F}_{2}$ or $F=\mathbf{F}_{2}(t)$ with $t$ transcendental over $\mathbf{F}_{2}$. Then the hyperbolic quadratics form over $F$ are 2-algebraic.

Proof. We only need to find an extension $E$ of $F$ such that $T_{E / F}$ is hyperbolic. We first remark that $p(x):=x^{4}+x^{3}+1$ is irreducible over $\mathbf{F}_{2}$ and also over $\mathbf{F}_{2}(t)$. Let $\alpha$ be a root of $p$ and $E=F(\alpha)$. We decompose the trace form $T_{E / F}$ with respect to the basis $\left\{\alpha, 1+\alpha^{3}\right\} \cup\left\{\alpha^{2}, \alpha+\alpha^{2}+\alpha^{3}\right\}$. Noting that this basis has the elements conjugate to $\alpha$, it is easy to recognise that each vector basis is isotropic, and furthermore by (2.1) we see that it is a symplectic basis. Hence, the space is hyperbolic. 
Theorem 4. Let $F$ be a field. If there exists $a \in F^{*}$ and $n$ odd such that the polynomial $x^{n}-a$ is irreducible over $F[x]$, then the hyperbolic quadratics space over $F$ are 2-algebraic.

Proof. Let $E=F(\alpha)$, where $\alpha \in \bar{F}$ and $\alpha^{n}=a$. For $1 \leq k \leq n-1$, the linear transformation $f_{\alpha^{k}}: x \mapsto x \alpha^{k}$ is given by the matrix $c_{i j}(k)$, where

$$
c_{i j}(k)= \begin{cases}1 & \text { if } j=i-k \\ a & \text { if } j=n+i-k \\ 0 & \text { otherwise }\end{cases}
$$

Then for $1 \leq k \leq n-1, \alpha^{k} \in E_{0}$, because $c_{i i}(k)=0$ for each $i$. Noting that $T_{1}(a)=a$ we obtain the decomposition

$$
E_{0}=\left\langle\alpha, \alpha^{n-1}\right\rangle \perp\left\langle\alpha^{2}, \alpha^{n-2}\right\rangle \perp \cdots \perp\left\langle\alpha^{\frac{n-1}{2}}, \alpha^{\frac{n+1}{2}}\right\rangle,
$$

where $\langle x, y\rangle$ is the space generate by $x$ and $y$. Hence, using that $n \neq 2 k$, we deduce that $T_{2}\left(\alpha^{k}\right)=0$ for $1 \leq k \leq n-1$,so $\left(E_{0}, T_{2}\right)=\left(\frac{n-1}{2}\right) \mathbf{H}$.

\section{References}

[BM] Bergé, A.M., Martinet, J.: Formes quadratiques et extensions en caractéristique 2, Sém. Théorie des Nombres de Bordeaux, exposé 35, (1982)-(1983).

[CP] Conner, P., Perlis, R.: A survey of trace forms of algebraic number fields, World Scientific, Singapur, (1984).

[EHP] Estes, R., Hurrelbrink, J., Perlis, R.: Total positivity and algebraic Witt classes, Comment. Math. Helvetici 60, pp. 284-290, (1985).

[KS] Krüspkeemper, M., Scharlau, W.: On trace forms of Hilbertian fields, Comment. Math. Helvetici 63, pp. 296-304, (1988).

[R] Revoy, P.: Remarques sur la forme trace, Linear and multilinear algebra 10, pp. 223-233, (1981). 
[Sa1] Sah, C.H.: Symmetric bilinear forms and quadratic forms, Journal of algebra 20, pp. 144-169, (1972).

[Se] Serre, J.P.: Linvariant de Witt de la forme $\operatorname{tr}\left(x^{2}\right)$, Comment. Math. Helvetici 59, pp. 657-676, (1984).

[Sch] Scharlau, W.: On trace forms of algebraic number fields, Math. Zeit. 127, pp. 125-127, (1987).

[Wat] Waterhouse, W.: Scaled trace forms over number fields, Arch. Math. 47, pp. 229-231, (1986).

Received : January, 2003.

\author{
Ana Cecilia de la Maza \\ Instituto de Matemática y Física \\ Universidad de Talca \\ Casilla 747 \\ Talca \\ e-mail : anace@inst-mat.utalca.cl
}

